\title{
An Automated Level Set Segmentation Approach for Lesion Detection in Dental Radiograph for Endodontic Treatment
}

\author{
Kavindra R. Jain \\ Department of Electronics and Communication \\ Ph D Scholar R K University Rajkot
}

\author{
N. C. Chauhan \\ Department Of Information Technology \\ A D Patel Institute of Technology, Anand, India
}

\begin{abstract}
Medical Imaging is advancing since inception. The engineering and technology with a wide variety of research in segmentation techniques have developed a wide research domain in the field of medical and bioinformatics. Application of segmentation techniques in medical areas for detection of abnormalities has made an add-on approach for both doctors and patients for prior diagnosis and proper treatment. Medical imaging in dental analysis is based on dental radiographs which help the medical practioners in locating hidden dental structures, malignant or benign masses, bone loss, and lesions. An important step during the analysis of dental imaging is extraction of decayed tooth from the dental radiographs. These digital dental radiograph plays a crucial role in detection and further diagnosis of decayed portion in jaw. In this paper, an automated segmentation method using multiphase level set approach is proposed for segmentation of dental radiograph and extraction of region of interest. The extracted region may provide better insight to the medical practitioners during their diagnosis. The results of the proposed segmentation method are analyzed qualitatively and quantitatively and are verified by experts of the domain for various categories.
\end{abstract}

\section{Keywords}

Image-Segmentation; Intensity-Inhomogeneity; Level Set; Root Canal Treatment (RCT); lesion.

\section{INTRODUCTION}

A digital dental radiograph reveals important information about the dental structure of the patient to dental practitioners. These radiographs are essential in oral diagnostic procedures and a good quality of radiograph with optimal image quality and visibility provides add on to it. One of the major challenges being faced in digital dental radiograph is in the segmentation of dental, gum and pulpal areas of the tooth. As the decayed lesions lie within it. The detection and further analysis of these lesion is quiet important for practioners as well as patient. Perseverance and accuracy by which these lesions must be identified is quiet crucial. Fragmentation of such medical images is most difficult task due to adverse topologies, the intricacy of medical structures and poor image quality due to noise, low contrast, similarity in body tissues, some sort of artifacts and limitations in scanning methods. Once this segmentation is done automatically then major part of the disease can be diagnosed and further treated. The crucial role over here is solely dependent on doctor's diagnosis to those radiographs which seems to be quiet varying from individual to individual. So the need of hour is to provide a clear region of interest from the given digital dental radiograph image. The authors are here by proposing an automatic approach for segmentation of individual tooth in dental radiographs to provide a clear insight of lesions and would help them in further treatment of patient. Since time being various segmentation methods in this domain have been proposed which can be broadly divided in two major categories. The first one is selection points based where an expert interaction is involved known as semiautomatic technique. In this method, medical practioners can select the seed points based on their knowledge and severity areas of radiograph to extract the region of interest. The major pitfall of this approach are firstly selection of seed points may vary from one to another. Secondly for more than one region of interest in a single radiograph the task would be quiet repetitive and bulky. The second method is automatic segmentation of dental radiographs images based on intensity variations. A popular category of automatic segmentation process is deformable contour method (DCM) which include edge based and region based segmentation techniques. To incorporate powerful and useful concepts of energy minimization, force, velocity and curve constraints, DCM based solutions are highly analytical and involve extensive numerical computations.

In this approach the user describes an initial guess for the contour, which is then converged by image driven forces to the boundaries of the desired objects. In such models, two dissimilar forces are measured - the internal forces, defined within the curve, are planned to keep the model smooth during the distortion process, while the external forces, which are computed from the underlying image data, are welldefined to move the model near an object edge or other anticipated features within the image [1].

Endodontic treatment ("endo" - inside; "dont" - tooth), commonly known as root canal treatment (RCT), is needed when the pulp becomes inflamed or infected. The causes could be deep tooth decay, repeated dental procedures on one tooth (replacing a large filling, for example), or traumatic damage such as a crack, chip or even a root fracture. Gum disease can also give rise to root canal problems necessitating root canal treatment [7].

Any of these issues can result in acute inflammation of the pulp, which causes swelling and pressure inside the tooth (that has nowhere to go), leading to tooth pain and, eventually, irreversible damage to the pulp. Once the pulp dies, the pain may subside initially, sometimes to return as an acute (painful) infection spreading into the periapical tissues ("peri" - around; "apex" - end), particularly the bone. It could also become a chronic (long-standing) infection with symptoms ranging from mild to severe [11].

To understand this further lets first of all know what is pulp. A space inside the tooth called the pulp chamber houses the root canal system and pulp living tissue that keeps the tooth 
vital (alive). The pulp includes blood vessels, nerves and connective tissues, and, during childhood, creates the surrounding hard tissues of the tooth. The general dental treatment involves removing the damaged area of the tooth (the pulp), dressing and disinfecting it and then filling and closing it [2]. Root canals alter from individual to individual and from tooth to tooth. That is why whenever an endodontic intermediation is planned, the foremost thing is accurate detection of root canal. Current medical imaging devices make it possible to record high resolution cross area of the teeth, which can be further utilized by image processing techniques to precisely locate the shape of the root canal. In literature, this problem has been addressed in several ways [12]. In this paper, we present an automatic segmentation based on level set technique to find the shape of damaged area in tooth.

Level set techniques perform numerical calculations including bends and surfaces to catch dynamic interfaces and state of art without the interest to parameterize the focuses on theories of bends and surfaces [4]. Level set details additionally minimize a target energy capacity which is obtained from the portrayal of picture information to accomplish picture division. An essential point of preference of level set strategies is that they can connote shapes of confounded topology and can deal with topological changes, for example, partitioning and combining, in a characteristic and proficient way, which is not permitted in parametric dynamic form models aside from additional circuitous ways which are introduced in the executions. Another method for level set techniques is that numerical calculations can be performed on a settled Cartesian lattice without having to parameterize the articles on a shape as in parametric dynamic form models.

This paper focuses on the diagnosis and detection of endodontic treatment using optimal bias corrected automatic segmentation technique. Further sections of this paper are as follows. Second section comprises of problem definition in dental radiograph. The third section discusses the background of aforesaid problem. Section 4 proposes a methodology being used along with the materials of automatic segmentation for the proper diagnosis and detection of decayed tooth. Section 5 concentrates on the results and discussion along with conclusion in section 6 .

\section{PROBLEM DEFINITION}

On the basis of radiograph taken the need of the hour is to fragment the image and look into the damaged areas of tooth individually. As shown in figure 1 the first part of the image represent a normal tooth which can be classified in three regions namely crown (above the gum area), neck (between crown and root portion), third one is root part. The other half of the image contains cavity or lesions rather, if the decay in tooth is in crown (enamel) or neck (dentin) part then capping can be done after removing the decayed portion. But if the decay is in side neck or roots (pulp) then RCT is suggested.

RCT is not recommended in the conditions where bacterial growth is not in pulp area. If a tooth has become abscessed, it may lead to a root canal. An abscess forms when the pulp of the tooth dies and a pus pocket forms around the end of the root. If the damaged area is in crown than only capping is recommended and if the damaged area in pulp is observed than RCT is suggested. Basically the routine extraction process of decayed tooth involves tooth extraction using forceps which is visible in dental radiograph image and this may lead to further cleaning of the roots to avoid further decayed of neighborhood teeth.

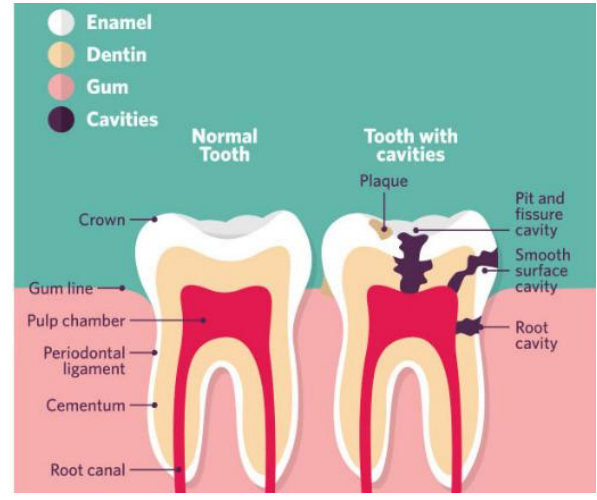

Figure 1. Normal Tooth and Tooth with cavities at crown, neck and root part [Courtesy by: - Dr. Ronak Panchal, Anand, India]

Thresholding based methods usually fail to discriminate between tooth and bone areas as their intensities are more or less similar. Image segmentation is a significant and a challenging task for dental radiographs as the pulp and gum areas fall under the same intensity levels. However the main motive behind the problem is to generate an automatic technique helpful for medical practioners in diagnosing the dental caries in more accurate way. To overcome that problem, the authors are suggesting extraction of tooth contour as a feature because they remain more invariant over time compared to other features of teeth. This extraction of teeth from a dental radiograph is not only automatically done but no initial seed points needs to be selected. Later we have utilized statistical and morphometric based mathematical calculation of the affected dental caries. Finally the aim is to help the dentists' visibility on detecting the abnormality on the dental intra-oral radiography.

\section{BACKGROUND}

Let $O$ be the image domain and $P: O \rightarrow \Re$ be a gray level image. While fragmenting the image $P$ using contour $D$ which separates the image domain $O$ into disjoint regions $O_{1}, O_{2}$, $O_{3} \ldots O_{N}$ and a piecewise smooth function $v$ that fulfilled image $P$ and is smooth inside each region $O_{j}$. Hence it can be formulated using Mumford Shah function [5].

$$
F^{M S}(v, D)=\int_{O}\left(P-v^{2}\right) d x+\mu \int_{O / D}\left|\nabla v^{2}\right| d x+v|D|
$$

Where $|D|$ stands for contour extent, on other side of the equation the first term is the value term which keeps $v$ close to $P$. The next is the smoothening term which forces $v$ to be smooth within each of the regions separated by $D$. The last one is to regularize contour $\mathrm{D}$.

Let $O_{1}, O_{2}, O_{3} \ldots O_{N}$ be the regions in $\mathrm{O}$ separated by $D$

Contour D is expressed as union of boundaries of regions denoted by

$$
\frac{O}{D}=\cup_{i=1}^{N} O_{i}
$$

Hence the above energy written in equation 1 can be modified as

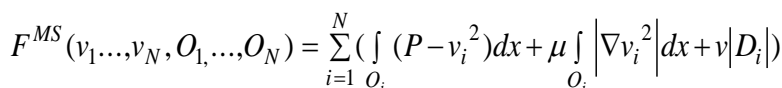

Where $v_{i}$ is a smooth function defined in the region $\mathrm{O}_{i}$. The approach to minimize this energy is called piecewise smooth model. The variables of the energy $\mathrm{F}^{\mathrm{MS}}$ include $\mathrm{N}$ different 
functions $v_{l}, \ldots . v_{N}$. The smoothness of every function is to be ensured by imposing a smoothing term $\mu \int_{O_{i}}\left|\nabla v_{i}{ }^{2}\right| d x$ in the functional $\mathrm{F}^{\mathrm{MS}}$. N partial differential equations are taken into considerations for elaborating $\mathrm{v}_{1} \ldots \ldots \mathrm{v}_{\mathrm{N}}$ associated with smoothening terms so introduced at each step in the contour D of regions $O_{1}, O_{2}, O_{3} \ldots O_{N}$. The result so obtained is quiet time consuming and not even accurate so moving further ChanVese model [3] is implemented with modification as,

$$
\begin{gathered}
F^{C V}\left(\theta, y_{1}, y_{2}\right)=\int_{O}\left|P(x)-y_{1}\right|^{2} H(\theta(x)) d x \\
+\int_{O}\left|P(x)-y_{2}\right|^{2}(1-H(\theta(x))) d x \\
+v \int_{O}|\nabla H(\theta(x))| d x
\end{gathered}
$$

where $H$ is the Heaviside function. $\theta$ is the level set function and $D$ is the zero level contour $\{\mathrm{D}=\{\mathrm{x}: \theta(\mathrm{x})=0\}\}$. It is now segmenting the image domain $\mathrm{O}$ into two disjoint regions $\mathrm{O}_{1}=\{\mathrm{x}: \theta(\mathrm{x})>0\}$ and $\mathrm{O}_{2}=\{\mathrm{x}: \theta(\mathrm{x})<0$. The first two terms in equation (2) are data fitting terms while the last one is weight functions $v>0$. Fragmentation of image simply occurs by $\theta, \mathrm{y}_{1}$ and $\mathrm{y}_{2}$ considering a fact that the image can be approximated by $\mathrm{y}_{1}$ and $\mathrm{y}_{2}$ in the regions $\mathrm{O}_{1}$ and $\mathrm{O}_{2}$ respectively.

\section{MATERIALS AND METHODS}

\subsection{Suggested Approach for Endodontic Treatment}

Keeping in mind the end goal to manage power inhomogeneities in image segmentation, we define our strategy in light of a image model that depicts the structure of true images, in which force inhomogeneity is credited to a part of an image. In this paper, we consider the accompanying multiplicative model of force inhomogeneity. From the prospect of image processing an image can be modeled as

$$
P=e L+r
$$

Where $\mathrm{L}$ indicates true or actual image. $e$ represents variation in intensity and $\mathrm{r}$ represents Gaussian noise. In previous section, we had mentioned that $P: O \rightarrow \Re$ in continuous time domain $O$. $e$ can be more profoundly defined as a constant at each point in the image. $L$ on the contrary can be considered as constants alongside of impaired sections of $O_{l}$, $\mathrm{O}_{2}$, and $\mathrm{O} 3 \ldots \mathrm{O}_{\mathrm{N}}$. Hence, according to (3) and above defined terminologies, we suggest a method to fragment the image based on the segmentation properties. A grid of $X$ (bias field) into subsets $X_{1}, X_{2}, X_{3}, \ldots, X_{N}$ must satisfy the following conditions, where $P\left(R_{i}\right)$ is a uniformity predicate for all elements in set $R[22,26]$ :

1) $U_{i=1}^{n} X_{i}=X$

2) For $i$ and $j$, if $i \neq j, X_{i} \cap X_{j}=\varphi$

3) $P\left(X_{i}\right)=$ TRUE for all $i$

4) $P\left(X_{i} \cup X_{j}\right)=$ FALSE if $i \neq j$

\subsection{Nearby Power Bunching Property}

Local level image division techniques normally depends on a specific local descriptor (e.g. force mean or a Gaussian dispersion) of the intensities in every area to be sectioned. In any case, it is difficult to give such area descriptor for pictures with force inhomogeneity. In addition, power inhomogeneity regularly prompt cover between the appropriations of the intensities in the areas $O_{1}, O_{2}, O_{3} \ldots O_{N}$. Along these lines, it is unthinkable to portion these locales straightforwardly taking into account the pixel intensities. Moreover, the property of neighborhood intensities is basic, which can be successfully abused in the definition of our technique for image fragmentation with synchronous estimation of the inclination field.

In light of the image model in (3) and the terminologies of $L$ and $e$, we can infer a helpful property of neighborhood intensities, which is alluded to as a nearby power grouping property as portrayed and justified underneath. To be specific, we consider a round neighborhood with a span focused at every point, $y \in O$ defined by $A_{y} \stackrel{\nabla}{=}\{x:|x-y| \leq \rho\}$. For a slowly varying bias field e, the values e $(\mathrm{x})$ for all $\mathrm{x}$ in the circular neighborhood $\mathrm{A}_{\mathrm{y}}$ are close to e (y),i.e.

$\mathrm{e}(\mathrm{x}) \approx \mathrm{e}(\mathrm{y}) \quad$ for $x \in A_{y}$

Therefore the intensities e (x) $L(x)$ in each sub region $A_{y} \cap O_{i}$ are close to constant $\mathrm{e}(\mathrm{y}) \mathrm{c}_{\mathrm{i}}$ i.e.

$\mathrm{e}(\mathrm{x}) \mathrm{L}(\mathrm{x}) \approx \mathrm{e}(\mathrm{y}) \mathrm{c}_{\mathrm{i}}$ for $\mathrm{A}_{\mathrm{y}} \cap \mathrm{O}_{\mathrm{i}} \ldots .(5)$

Hence the modified image model in equation (3) becomes

$$
P(x)=e(y) c_{i}+r(x) \quad \text { for } x \in A_{y} \cap O_{i}
$$

Where $r(x)$ is additive zero mean Gaussian noise. Hence the intensities in the set

$$
P_{y}^{i}=\left\{P(x): x \in A_{y} \cap O_{i}\right.
$$

Ensembles in such a way that every distinct bunch are well separated and have their own loci as $\mathrm{m}_{\mathrm{i}} \approx \mathrm{e}(\mathrm{y}) \mathrm{c}_{\mathrm{i}}$. Each such loci is unique in itself and have its own identity. This property of nearby bunching is used further in our proposed approach.

\subsection{Vitality Detailing}

In the upper subsection the nearby power bunching capability helps us in getting a clear idea that the intensities in the nearby areas can be distinguished into $N$ such bunches having their own loci. With the help of $K$-means method our target is to reduce the number of bunches. It can be done using a windowing function. So the final equation for such bunching criteria can be

$$
\varepsilon_{y}=\sum_{i=1}^{N} \int_{O_{i}} K(y-x)\left|P(x)-e(y) c_{i}\right|^{2} d x
$$

Where $\varepsilon$ represents the vitality, $K(y-x)$ expresses the windowing function. Therefore the need is to reduce the vitality for all $y$ in $O$. This can be done by reducing the integral of vitality with respect to $O$.

\section{LEVEL SET DEFINITION AND VITALITY DETAILING}

The vitality $\varepsilon$ in (6) is communicated as $O_{1}, O_{2}$, and $O 3 \ldots O_{N}$. It is difficult to determine an answer for the vitality minimization issue from this statement of $\varepsilon$. In this area, the vitality is changed over to a level set detailing by speaking to the disjoint locales $O_{1}, O_{2}, O_{3} \ldots O_{N}$ with various level set capacities, with a regularization term on these level set capacities. In the level set detailing, the vitality minimization can be settled by utilizing entrenched variational techniques 
[6]. In level set techniques, a level set capacity is a capacity that take positive and negative signs, which can be utilized to speak to a segment of the area $O$ into three disjoint districts $O_{1}, O_{2}$ and $O_{3}$ Let $P: O \rightarrow \Re$ be a level set capacity, then its signs define three disjoint locales,

$$
\mathrm{O}_{1}=\{\mathrm{x}: \theta(\mathrm{x})>0\}, \mathrm{O}_{2}=\{\mathrm{x}: \theta(\mathrm{x})<0\} \text { and } \mathrm{O}_{3}=\{\mathrm{x}: \theta(\mathrm{x})=0\} \text { (7) }
$$

Which frame a parcel of the space $O$. For instance two or more level set capacities can be utilized to areas of $O$. The level set definition of the vitality can then be defined as three phase level set formulation in next subsection.

\subsection{Three Phase Level Set Formulation}

We first consider the three phase case [7]: the region of interest $O$ is divided into three disjoint locales $O_{1}, O_{2}$ and $O_{3}$. Furthermore for this situation, a level set capacity $\theta$ is utilized to represent three regions based on equation (7). These regions can now be defined by two level sets as $\theta_{1}$ and $\theta_{2}$ respectively, where $M_{1}\left(\theta_{1}, \theta_{2}\right)=H\left(\theta_{1}\right) H\left(\theta_{2}\right), M_{2}\left(\theta_{1}, \theta_{2}\right)=$ $H\left(\theta_{1}\right)\left(1-H\left(\theta_{2}\right)\right)$ and $M_{3}\left(\theta_{1}, \theta_{2}\right)=1-H\left(\theta_{1}\right)$ respectively, where $H$ represents Heaviside function. Thus the energy equation (6) can be modified as

$\varepsilon=\int\left(\sum_{i=1}^{N} \int K(y-x)\left|P(x)-e(y) c_{i}\right|^{2} M_{i}\left(\theta_{1}(x), \theta_{2}(x)\right) d x\right) d y{ }^{(8)}$

The vitality detailing is achieved by a continuous process, in each iteration the vitality is reduced based upon the $\varepsilon(\theta, e, c)$ with respect to each variable $\theta, e, c$. We can rewrite this equation in the following form:

$\varepsilon(\theta, e, c)=\int\left(\sum_{i=1}^{N} \int e_{i}(x) M_{i}(\theta(x)) d x\right) \cdots$

Where $e_{i}$ is the function defined by

$e_{i}(x)=\int K(y-x)\left|P(x)-e(y) c_{i}\right|^{2} d y \cdots($

With the help of above equation we try to minimize this energy and form an equation on the basis of level set function $\theta$ and estimation of the bias field e. the process of minimization is iterative in nature one is able to minimize $\mathrm{F}(\theta$ ,c,e) with respect to each of the variable $\theta, \mathrm{c}$ and e. So the solution to vitality reduction is with respect to each of the variables as follows:-

\subsubsection{Vitality detailing with respect to $\theta$ :}

For fixed values of $e$ and $c$ the detailing of $\varepsilon(\theta, e, c)$ can be calculated using gradient descent method by gradient flow equation

$$
\frac{\partial \theta}{\partial t}=-\frac{\partial F}{\partial \theta}
$$

Where $\frac{\partial F}{\partial \theta}$ is the Gateaux derivative which can further be modified as

$$
\begin{gathered}
\frac{\partial \theta}{\partial t}=- \\
\delta(\theta)\left(e_{1}-e_{2}\right)+v \delta(\theta) \operatorname{div}\left(\frac{\nabla \theta}{|\nabla \theta|}\right) \\
+\mu \operatorname{div}\left(d_{p}(|\nabla \theta|) \nabla \theta\right) \cdots(11)
\end{gathered}
$$

Where gradient and divergence operator along with $d_{p}$ is defined as

$$
d_{p}(s) \stackrel{\Delta}{=} \frac{p^{\prime}(s)}{s}
$$

as are merged altogether as explained in [11], can be used for level set evolution. During this process the vitality $\varepsilon(\theta, e, l)$ is detailed with respect to $e$ and $l$.

\subsubsection{Vitality detailing with respect to $c$ :}

For the constant values of $\theta$ and $e$ the $l$ parameter minimizes

the vitality $\varepsilon(\theta, e, l)$ denoted by $\hat{c}=\hat{c}_{i}, \ldots . ., \hat{c}_{n}$ is symbolized as,

$$
\hat{c}_{i}=\frac{\int(e * M) P v_{i} d y}{\int\left(e^{2} * M\right) v_{i} d y}
$$

as $u_{i}(\mathrm{y})=\mathrm{M}_{\mathrm{i}}(\theta(\mathrm{y}))$

\subsubsection{Vitality detailing with respect to e:}

For the constant values of $\theta$ and $c$ the $e$ parameter minimizes the vitality $\varepsilon(\theta, e, c)$ denoted by $\hat{e}$ is provided by equation (13). The convolution in (9) shows the slowly varying characteristics of $\hat{e}$.

$$
\hat{e}=\frac{\left(P \sum_{i=1}^{N} l_{i} v_{i}\right) * M}{\left(P \sum_{i=1}^{N} l_{i}^{2} v_{i}\right) * M}
$$

Moreover the Heaviside function $\mathrm{H}$ is swapped by a suave function that provides a close value to $\mathrm{H}$.

\subsection{Multiphase Level Set Formulation}

For the case of $\mathrm{N} \geq 3$ or more and notational simplicity these level set functions $\left(\theta_{l}, \theta_{2} \ldots . \theta_{k}\right)$ by a vector valued function $\Phi$. Hence the membership function can be reformulated as $M_{i}(\Phi)$. The energy can be converted to multiphase level set formulation as

$$
\varepsilon(\Phi, e, c)=\int\left(\sum_{i=1}^{N} \int e_{i}(x) M_{i}(\Phi(x)) d x\right)
$$

The energy functional $\mathrm{F}$ in our multiphase level set function formulation based on gradient flow equation (11) can be further formulated staring from $\theta_{1}, \theta_{2} \ldots . \theta_{k}$ form individual gradient flow equation.

\section{RESULT AND DISCUSSION}

To understand the results of our proposed method for fragmentation at an initial level, we had taken 80 radiographic images with the consult of doctor and patient. These radiographic images are DICOM images of dimensions $(1200 * 1600)$ each with $372 \mathrm{~KB}$ size. For instance to be considered a radiographic image as shown in figure 2 which depicts the problems being faced for endodontic treatment is taken. According to our suggested approach first of all the image undergoes through preprocessing and then after segmentation. In this method, random points for initialization are being set and then slowly these areas grow according to the vitality increase or decrease in the nearby regions based on the mathematical model described in above section. Finally, we get a fragmented image based on three phase level set method as described in section IV. The two regions so depicted clearly represents the regions of interest as the red 
region so displayed clearly shows the affected areas for endodontic treatment as shown in figure 3. Later on based on fragmentation and level set the three areas are given same vitality for the purpose of identification as black grey and white. Hence, as shown in figure 4 the black portion between two teeth are the affected areas for analysis along with the tooth itself having black portion in neck of tooth as encircled in figure 4 . The same process was applied to several other dental radiographs and result were quiet effective as shown in figure 5 .

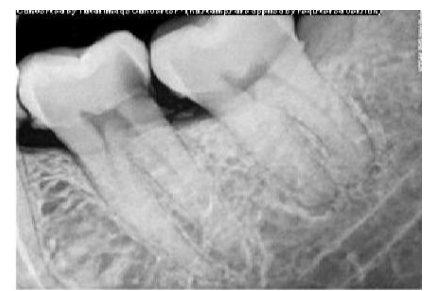

Figure 2 A sample Dental Radiograph [Courtesy by Dr. Ronak Panchal]

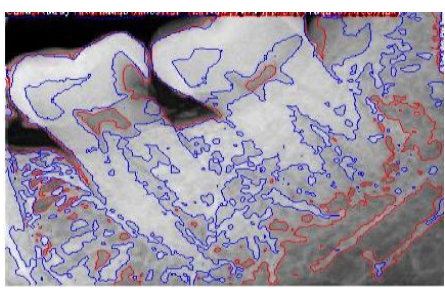

Figure 3 Three phase level set segmentation (after 99 iterations

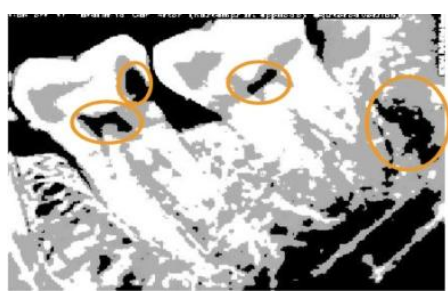

Figure 4 final segmented image

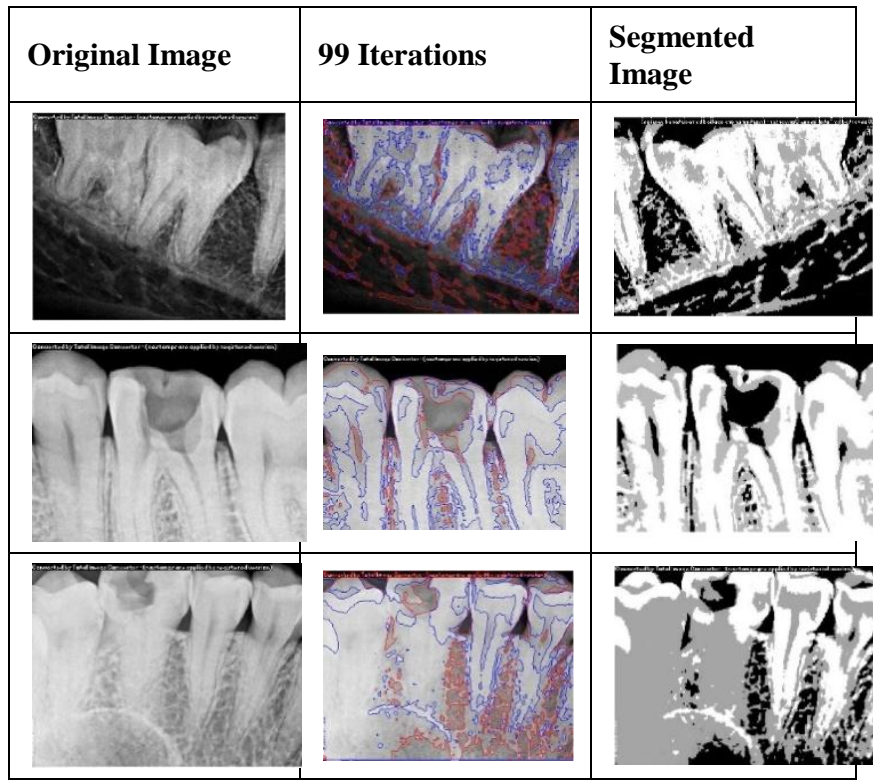

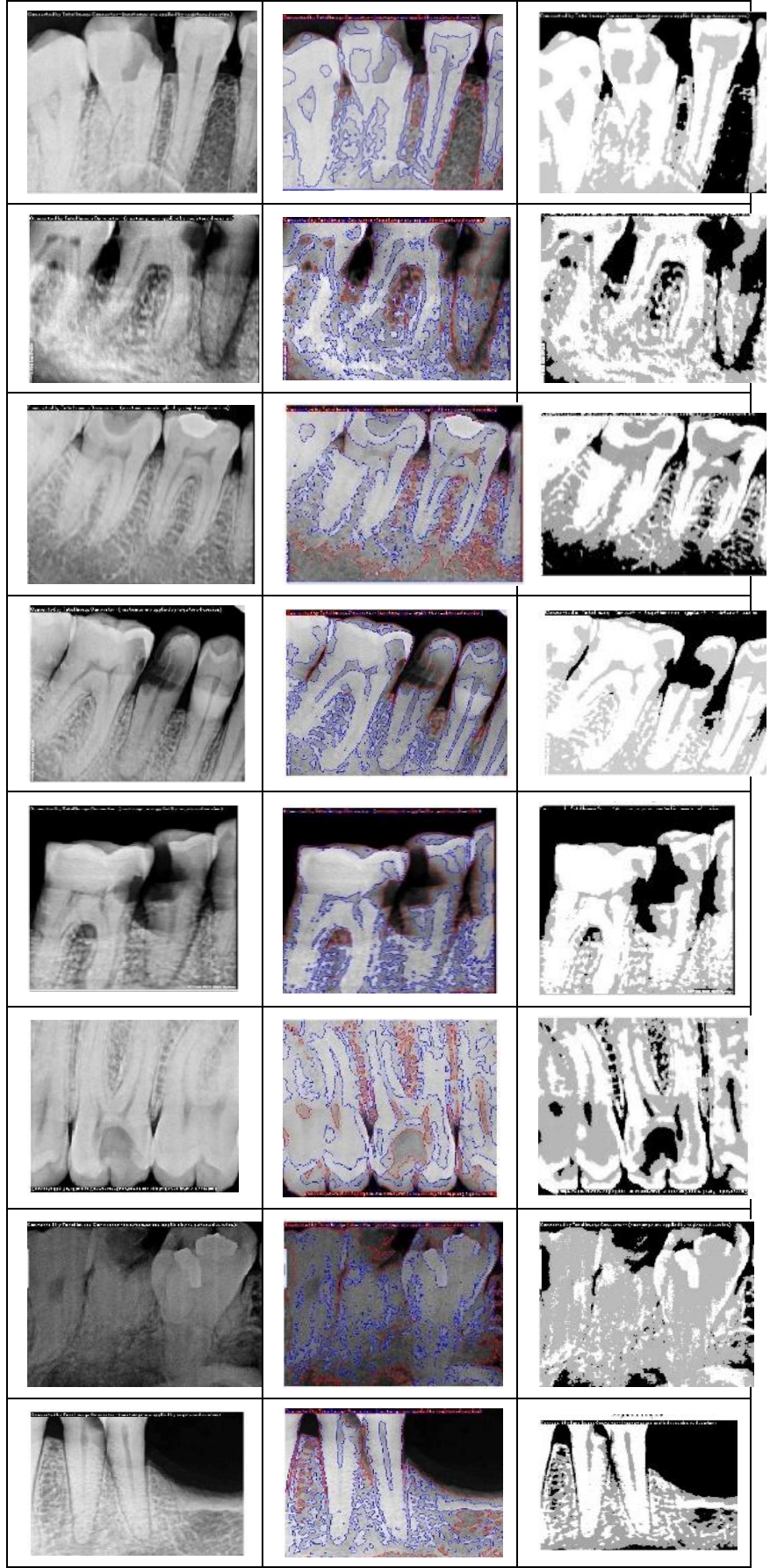

Figure 5: Results of segmentation on different images from radiograph image dataset. (The first column represents actual radiograph, the middle column represents intermediate results after 99 iterations, and the last column shows final segmentation results.)

The results were applied on 96 such database images. The results so obtained were further analyzed for the purpose of accuracy according to the dental experts.

\subsection{Evaluation Measurements for}

\section{Prediction of Endodontic Treatment}

In this section, we focus on identifying performance of proposed method in prediction of the endodontic treatment. The evaluation measurements include accuracy (recognition rate), sensitivity (recall), specificity and precision. [Before the measurement starts it is required to understand that the 
resultant output of our algorithm is for the help of doctor in further process of treatment so the final decision being taken is only by him or her. For the sake of convenience we went to two dentist and showed them the original image. Based on the expert advice by the dentist we requested to mark the ROI for the given radiographic images.

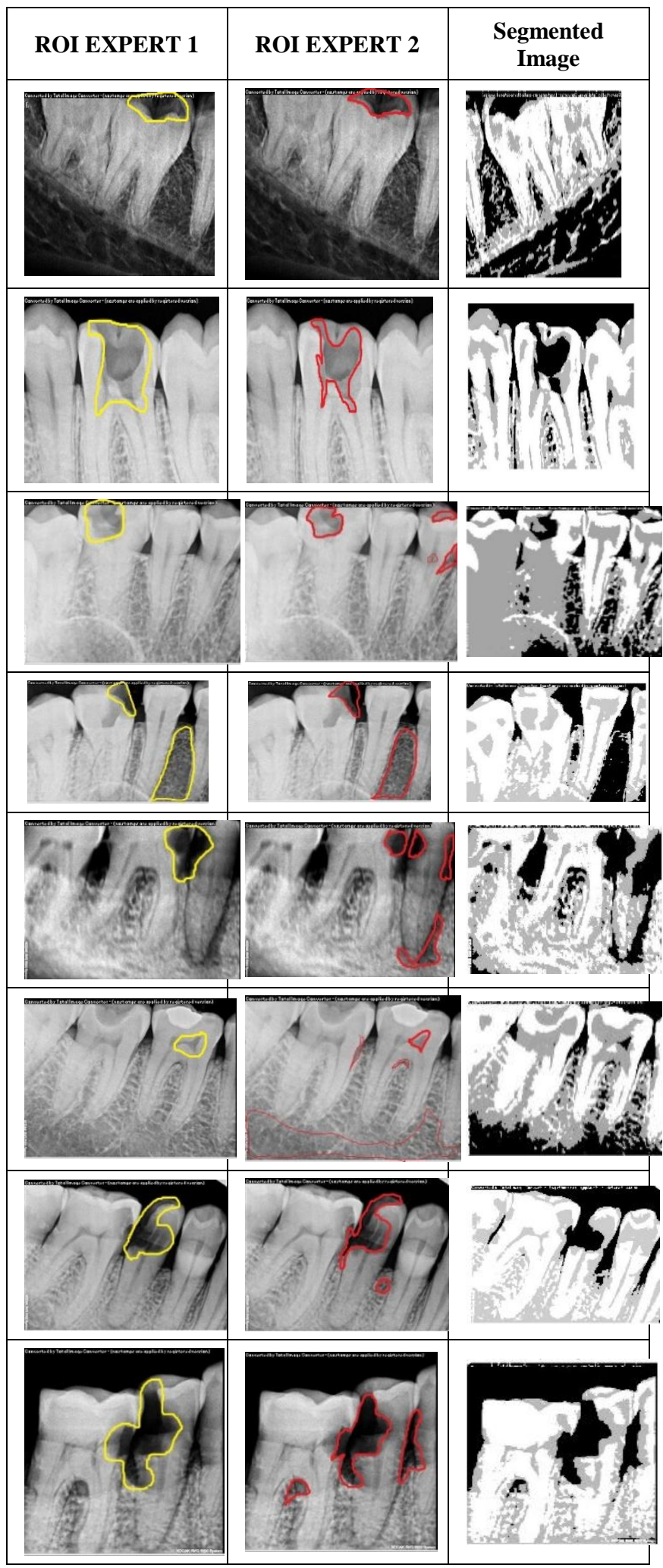

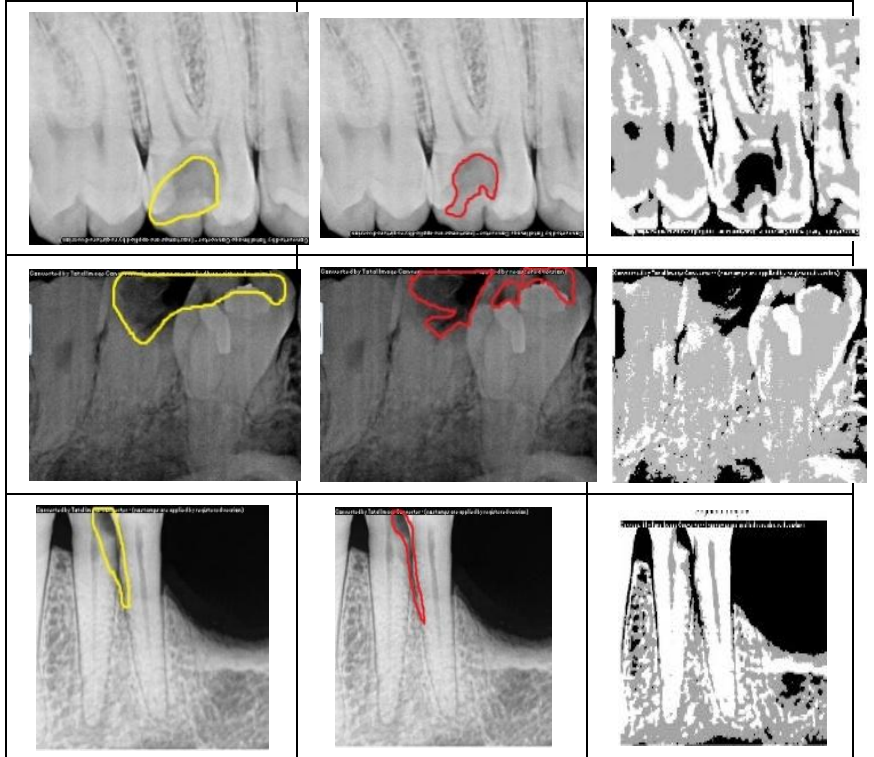

Figure 6: Comparison of Segmented image results with the ROIs marked by two domain experts. The first column represents ROI indicated by first expert, the second column represents ROI indicated by second expert, and the third column indicates the segmented image by the proposed method.

As shown in figure 6, the first column contains images in which ROI is marked by first expert i.e. the ROI is selected by one dentist in yellow boundaries. The second column represents images in which ROI is indicated by second expert i.e. the ROI is selected by another dentist in red boundaries. The third column represents the segmented result by our proposed algorithm. In order to measure the performance of the method, we describe few terminologies like positive region (pixels of the main class of interest) and negative region (region of image except positive region). More specifically, the positive region means the ROI according to domain expert (dentist) in our radiographic image and false region means the areas excluding ROI. Let $P$ be the number of pixels in positive region and $N$ be the number of pixels in negative region. For each region, we compare the classifier's predicted class label with the region's known class label. The four important terminologies used in the whole process of measurement are as follows. $[23,24]$

True Positives (TP):- These refer to the positive region(s) that was/were correctly labeled by the classifier.

True Negatives (TN):- These refer to the negative region(s) that was/were correctly labeled by the classifier.

False Positive (FP):- These refer to the negative region(s) that was/were incorrectly labeled by the classifier as positive region(s)

False Negative (FN):- These refer to the positive region(s) that were incorrectly labeled by the classifier as negative region(s).

The above terms are summarized in a confusion matrix as shown in table 1 where the predicted class means the segmented region(s) of affected tooth based on proposed algorithm and the actual class means the ROI of expert 1. In a similar fashion for ROI by expert 2 with the segmented results is also calculated. 
Table 1:- Confusion Matrix

\begin{tabular}{|l|l||l|l|l|}
\hline & \multicolumn{4}{|c|}{ Predicted Class } \\
\hline \multirow{4}{*}{$\begin{array}{l}\text { Actual } \\
\text { class }\end{array}$} & \multicolumn{1}{|c|}{ YES } & NO & TOTAL \\
\cline { 2 - 5 } & \multirow{2}{*}{ YES } & TP & FN & P \\
\cline { 2 - 5 } & NO & FP & TN & N \\
\cline { 2 - 5 } & TOTAL & $\mathrm{P}^{\prime}$ & $\mathrm{N}^{\prime}$ & $\mathrm{P}+\mathrm{N}$ \\
\hline
\end{tabular}

Based on the terms mentioned in confusion matrix, three performance evaluation measures - accuracy, error rate, and specificity are defined as follows.

Accuracy (A): On a given data set it is defined as the percentage of test pixels/regions that are correctly classified by the classifier. It is given by the following formula

$$
\text { accuracy }=\frac{T P+T N}{P+N}
$$

Error Rate (ER): On a given data set it is defined as 1Accuracy. It is given by the following formula

$$
\text { error rate }=\frac{F P+F N}{P+N}
$$

Precision (PR): On a given data it refers to as true positive pixels/regions that are identified by exactness.

$$
\text { precision }=\frac{T P}{T P+F P}
$$

Specificity (S): On a given data set it is defined as the true negative pixels/regions that are correctly identified. It is given by the following formula

$$
\text { specificit } y=\frac{T N}{N}
$$

Based on the above defined parameters for measurement, the confusion matrix for one of the radiographic images and ROI of expert-1 as shown in table 2 .

Table 2:- Confusion matrix of a sample image for ROI by expert 1

\begin{tabular}{|c|c||c|c|c|}
\hline & \multicolumn{4}{|c|}{ Predicted Class } \\
\hline \multirow{4}{*}{$\begin{array}{c}\text { Actual } \\
\text { class }\end{array}$} & \multicolumn{1}{|c|}{ YES } & NO & TOTAL \\
\cline { 2 - 5 } & $\boldsymbol{Y E S}$ & 791 & 388 & 1179 \\
\cline { 2 - 5 } & NO & 388 & 66306 & 66694 \\
\cline { 2 - 5 } & TOTAL & 1179 & 66694 & 67873 \\
\hline
\end{tabular}

So according to the above confusion matrix the accuracy of our segmented result based on ROI expert 1 is $98.88 \%$, error rate is $1.14 \%$, precision is $67.09 \%$ and specificity is 99.4182 $\%$.The confusion matrix for the same radiographic image and ROI indicated by expert 2 is shown in table 3 .

Table 3:- Confusion matrix of the sample image for ROI indicated by expert 2

\begin{tabular}{|c|c||c|c|c|}
\hline & \multicolumn{4}{|c|}{ Predicted Class } \\
\hline \multirow{4}{*}{$\begin{array}{c}\text { Actual } \\
\text { class }\end{array}$} & \multicolumn{1}{|c|}{ YES } & NO & TOTAL \\
\cline { 2 - 5 } & YES & 791 & 39 & 830 \\
\cline { 2 - 5 } & NOT & 39 & 67097 & 67136 \\
\cline { 2 - 5 } & TOTAL & 830 & 67136 & 67936 \\
\hline
\end{tabular}

According to the confusion matrix shown in Table 2 and 3 , the accuracy, precision, error rate and specificity of our segmented result based on ROI expert 1 and expert 2 is presented in table 4 and 5 .
Table 4:- Confusion matrix of the sample image (used in table 2) for ROI indicated by expert 1

\begin{tabular}{|c|c|c|c|c|}
\hline Images & A & PR & ER & S \\
\hline 1 & 98.30 & 96.46 & 1.69 & 99.10 \\
\hline 2 & 95.71 & 78.28 & 4.28 & 97.59 \\
\hline 3 & 97.69 & 88.87 & 2.30 & 98.78 \\
\hline 4 & 97.81 & 88.31 & 2.18 & 98.77 \\
\hline 5 & 98.99 & 91.30 & 1.00 & 99.43 \\
\hline 6 & 86.75 & 83.04 & 3.24 & 96.48 \\
\hline 7 & 98.81 & 90.46 & 1.18 & 99.37 \\
\hline 8 & 93.45 & 63.39 & 6.54 & 96.32 \\
\hline 9 & 98.26 & 73.24 & 1.73 & 99.09 \\
\hline 10 & 98.85 & 93.35 & 1.14 & 99.37 \\
\hline 11 & 99.43 & 88.19 & 0.56 & 99.72 \\
\hline 12 & 99.85 & 94.12 & 0.14 & 97.31 \\
\hline 13 & 97.35 & 90.63 & 2.64 & 91.49 \\
\hline $\begin{array}{c}\text { Avg. 100 } \\
\text { images }\end{array}$ & $\mathbf{9 7 . 5 3}$ & $\mathbf{7 9 . 7 9}$ & $\mathbf{2 . 4 6}$ & $\mathbf{9 8 . 6 4}$ \\
\hline
\end{tabular}

Table 5:- Confusion matrix of the sample image (used in table 3) for ROI indicated by expert 2

\begin{tabular}{|c|c|c|c|c|}
\hline Images & A & PR & ER & S \\
\hline 1 & 98.12 & 74.05 & 1.87 & 98.98 \\
\hline 2 & 95.97 & 80.82 & 4.02 & 97.93 \\
\hline 3 & 97.65 & 67.70 & 2.34 & 98.73 \\
\hline 4 & 96.21 & 82.18 & 3.78 & 97.98 \\
\hline 5 & 98.28 & 85.73 & 1.71 & 99.05 \\
\hline 6 & 99.49 & 88.22 & 0.50 & 99.75 \\
\hline 7 & 99.90 & 99.39 & 0.09 & 99.96 \\
\hline 8 & 92.66 & 57.71 & 7.33 & 95.76 \\
\hline 9 & 95.40 & 49.64 & 4.59 & 97.53 \\
\hline 10 & 98.41 & 91.66 & 1.58 & 99.20 \\
\hline 11 & 98.81 & 79.17 & 1.18 & 99.46 \\
\hline 12 & 99.13 & 80.90 & 0.86 & 99.62 \\
\hline 13 & 94.18 & 81.47 & 5.81 & 96.45 \\
\hline Avg. & $\mathbf{9 6 . 0 0}$ & $\mathbf{6 6 . 1 2}$ & $\mathbf{3 . 9 9}$ & $\mathbf{9 7 . 8 2}$ \\
\hline 100 images & & & & \\
\hline
\end{tabular}

Hence, now with the help of results of performance measures, it is can be depicted that the suggested approach is quiet helpful for the automatic segmentation and analysis of dental radiograph images during the process of endodontic treatment. The results produced by the proposed method were shown to the respective dentist and were found to be really helpful. The average is resultant of 100 such images taken for analysis is shown in tables 4 and 5 .

\section{CONCLUSION}

In this paper, an automated segmentation method based on level set approach for segmenting region of interest from a dental radiographic image is presented. The results produced by the proposed method was compared with the ground truth results obtained with two domain experts. Based on the evaluation measures, the proposed method is found to be quite effective in identification of interested region. The proposed method can be useful for assistance to the dental medical practioners during their endodontic treatment of the tooth. The method can further be evaluated rigorously and can also be integrated in any computer based diagnostic tool.

\section{ACKNOWLEDGEMENT}

The authors of this paper express deep gratitude to Dr. Ronak Panchal - a dental practitioner at Anand, India for sharing image dataset and domain knowledge, and also for providing valuable feedback. 


\section{REFERENCES}

[1] Chunming Li, Rui Huang, Zhaohua Ding, J. Chris Gatenby, "A Level Set Method for Image Segmentation in the Presence of Intensity In-homogeneities With Application to MRI", in IEEE Transactions on Image Processing (Volume:20 , Issue: 7) ,pp. 2007 - 2016, April 2011

[2] D. Cremers, "A multiphase levelset framework for variational motion segmentation," in Proc. Scale Space Meth. Comput. Vis., Isle of Skye, U.K., Jun. 2003, pp. 599-614.

[3] C. Li, R. Huang, Z. Ding, C. Gatenby, D. Metaxas, and J. Gore, "A variational level set approach to segmentation and bias correction of medical images with intensity inhomogeneity," in Proc. Med. Image Comput. Comput. Aided Intervention, 2008, vol. LNCS 5242, pp. 10831091, Part II

[4] T. Chan and L. Vese, "Active contours without edges," IEEE Trans. Image. Process., vol. 10, no. 2, pp. 266277, Feb. 2001.

[5] L. Vese and T. Chan, "A multiphase level set framework for image segmentation using the Mumford and Shah model," Int. J. Comput. Vis., vol. 50, no. 3, pp. 271-293, Dec. 2002.

[6] I Ketut Eddy Purnama, Ima Kurniastuti, Margareta Rinastiti, Mauridhi Hery Purnomo, "Semi-Automatic Determination of Root Canal Length in Dental Dental radiograph Image.

[7] R. B. Tiwari, Prof. A. R. Yardi, "Dental Dental radiograph image enhancement based on human visual system and local image statistics"., International Conference on Image Processing, Computer Vision and Pattern Recognition, 2006, pp 100-108

[8] C. K. Modi , K. J. Pithadiya ,J. D.Chauhan, K. R. Jain, “ Comparative study of Optimal edge detection algorithms for liquid level inspection in Bottles", International conference on Emerging Trends in Engineering and Technology, pp 447-452, 2009.

[9] E. H. Said, D. E. M. Nassar, G. Fahmy, H. H. Ammar. "Teeth segmentation in digitized dental Dental radiograph films using mathematical morphology," IEEE Transactions on information forensic and security, vol. 1, Issue. 2, pp. 178-189, June. 2006.

[10] White \& Pharoah "Oral Radiology- Principles and Interpretation", Fifth Edition (2005), selected illustration by Dr. Donald O'Connor. ISBN 0-323-02001,published by MOSBY (An affiliate of Elsevier)

[11] Shafer's Tb. "Textbook of Oral Pathology" sixth edition, 2006.

[12] S.A.Ahmed, M.N.Taib, N.E.A.Khalid, R.Ahmad, H.Taib "Performance of compound enhancement algorithms on dental radiograph images" WASET-2011
[13] Stefan Michel, Saskia M.Koller, Markus Ruh, Adrian Schwaninger, "Do "Image Enhancement" Functions Really Enhance Dental radiograph Image Interpretation?" Cognitive Science Journal Archieve 2007

[14] Nirav P. Desai, D. B. Prajapati "A simple and novel CBIR technique for features extraction using AM dental radiographs" CSNT (IEEE) 2013, Gwalior, pp.198-202, 2012

[15] Maja Omanovic, Jeff J. Orchard "Exhaustive Matching of Dental Dental radiographs for Human Forensic Identification "Journal of the Canadian Society of Forensic Science, 2008

[16] S.Jadhav, R. Shriram "Dental biometrics used in forensic science" IJERS/Vol.III/ Issue I/January-March, 2012/2629

[17] Omaima Nomir, M.A.Mottaleb "A system for human identification from Dental radiograph dental radiographs" Pattern Recognition 38 (2005) 1295 - 1305.

[18] Sharifah Lailee, Nursuriati Jamil "Segmentation of Natural Images Using an Improved Thresholding-based Technique" IRIS 2012, Elsevier Procedia engg. Conference pp938-944.

[19] TM Lehmann, E.Troeltsch, K Spitzer" Image processing and enhancement provided by commercial dental software programs: A Technical Report" Dentomaxillofacial Radiology (2002) 31, 264-272.

[20] T. N. Cornsweet, Visual Perception, Academic Press, New York, 1970.

[21] Yan Gao and Jesse S. Jin, "Fast LoG Filtering using Recursive Filters", IEEE Trans., pp.133 -138,1995.

[22] Osher S, Fedkiw R (2006) Level set methods and dynamic implicit surfaces, vol 153. Springer Science \& Business Media.

[23] Malladi R, Sethian JA, Vemuri BC (1995) Shape modeling with front propagation: a level set approach. IEEE Trans Pattern Anal Mach Intell 17(2):158-175

[24] Weickert J, Kuhne G (2003) Fast methods for implicit active contour models. In: Geometric level set methods in imaging, vision, and graphics. Springer, pp 43-57.

[25] Shuo L, Fevens T, Krzyzak A, Li S (2006) An automatic variational level set segmentation framework for computer aided dental dental radiographs analysis in clinical environments. Comput Med Imaging Graph 30(2):65-74.

[26] Qu Y, Wong TT, Heng PA (2007) Image segmentation using the level set method. In: Deformable models. Springer, pp 95-122. 